\title{
A critical view on occlusion and registration
}

\author{
Ruth Nebel* \\ Independent Researcher, Germany
}

\begin{abstract}
"Occlusion" does not seem to be a sufficient parameter for diagnostics. In a similar way, "jaw relation" or "centric relation" do not seem to hit the point of the matter. In fact, they seem to be composed not of one, but of various parameters. Dental registration does not consider the parameters gravitation and spatial orientation, which contribute to the position of the jaws in the upright position. These parameters, however, are being processed in mandible and maxilla during motion in the environment. Discrepancies in the correct fit of prostheses are likely to arise from these unconsidered parameters in the upright posture of daily living. Hypothetically, discrepancies do not originate from the masticatory system, but from the postural system. Temporomandibular dysfunction hypothetically seems to develop from this very problem. Considering all of the acting parameters should improve diagnostics and treatment immensely.
\end{abstract}

\section{Introduction}

Dental diagnostics like "occlusion", "jaw relation" or "centric relation" do not seem to hit the point of the matter. Currently, registration is done in a $30^{\circ}$ dorsal position or any upright position. The mandible thereby is positioned, more or less guided, symmetrical to the maxilla. Additionally, sometimes is done a preliminary balancing of the chewing muscles. Obviously, this mode of registration considers the masticatory system only, assuming that the positioning of the jaws is a function of the chewing muscles only.

\section{Observations and hypotheses}

Consequently, prostheses are not always comfortable for the patient, although the occlusion proves to be according to the registered state. This is because the position of jaws during clenching (registration) differs from that in the upright posture while orientating in the environment. Problems with the registered state ("occlusion") hypothetically develop from the upright situation in daily living (orientating in the environment) under the influence of gravitation - this, however, is a function of the postural system. The present hypotheses offer a new perspective on that theme: Discrepancies are due to the postural function. The postural system AND the masticatory system are processing in jaw and teeth [1].

Clenching as function of the chewing muscles is done mostly symmetrical [2]. In the postural function, however, chewing and neck muscles typically are not symmetrical. They serve as a muscle frame which adapts passively to the positions of the bones they are fixed to. Temporary asymmetries physiologically occur during motion, while the mandible hangs or sways below the maxilla [3]. This, however, is no active "dysbalance", but reactive.

The postural system may cause a lateroflected (extended, flected) head posture - with the resulting angulation between the skull and the mandible [4]. This discrepancy hypothetically arises from the different references of maxilla and mandible:

\section{Spatial parameters in the jaws}

1. Maxilla and its teeth (chewing plane) hypothetically refer to an extracorporeal parameter: they are related to the true horizontal and the movement direction. This spatial alignment is kept even in motion. If the upper chewing plane is not transverse but rises to one side of the head, the head will consequently be lowered in this side. Hypothetically, this is done to realign the chewing plane to the true horizontal. Thereby, the NHP (natural head position) is changed. Problems may arise from this posture.

2. The mandible is related to gravitation: It hangs, as far as possible, according to the gravitational direction [5]. A further aspect is to mention concerning the import of mistakes from a current in the following prosthesis:

3. Asymmetries in shape and space of the prosthesis may force the tongue and the mandible into an unnatural position: If this prosthesis is worn with registration for a future prosthesis, these mistakes will be imported into the next prosthesis

\section{Ad 1: Orientation of the upper chewing plane to space}

Too high fillings, too long or too short crowns or bridges will change the course of the upper chewing plane to the skull $[4,6]$. Prosthetic "tooth" length usually differs from the original length. Accordingly, the level of the prosthetic undersurface changes the level of the chewing plane, which is the reference of the postural system. An oblique position of the upper chewing plane frequently occurs in patients with prostheses.

In the x-ray of figure 1, the prosthesis (bridge) in the upper left quarter is lacking about $3 \mathrm{~mm}$ in length compared with the original teeth length. Consequently, the chewing plane rises up to the left side of the skull, so that the distance between the chewing plane - and the orbita is smaller in the left than in the right hand side. The head shows a permanent lateroflexion to the left and a rotation to the right. This is a

Correspondence to: Ruth Nebel, Physician/Physiotherapist, Independent Researcher, Germany, E-mail: ruth.nebel@mail.de

Received: January 10 2017; Accepted: January 24, 2017; Published: January 26, 2017 
function of the postural system to lower the undersurface of the bridge in relation to spaceand to realign it to the true horizontal. Summing up, the oblique chewing plane seems to be the cause for the lateroflection. Changes in the level of the chewing-plane may be seen frequently in patients with TMD [7-9] and seem to cause "Temporomandibular dysfunction (TMD)".

\section{Ad 2: The mandible follows the gravitational vector}

The mandible hangs along gravity. If the head is positioned in a lateroflection, flection or extension, the mandible will hang at an angle to the skull [5]. This concerns the relation of the lower to the upper teeth as well.

The $\mathrm{x}$-ray in figure 1 shows the skull in a lateroflection to the left and a rotation to the right. The mandible hangs at an angle to the skull, the left ramus is partially hidden in the skull. The left condyle is shifted dorsal-cranial, the right one ventral-caudal in relation to the fossa (see to the role of condyle positioning [10-13].

Just in case of an upright head posture (= head's median runs according to the gravitational direction), the mandible will hang symmetric. The patient's bite will be symmetric in this case and there will be little discrepancy to the situation of registration. In each NHP, the head moves physiologically during motion. During walking, the mandible sways under the skull [3].

\section{The spatial articulator}

The spatial articulator (Figure 2) shows the teeth in their position during motion in the environment. In this example, the upper teeth in the right side are lacking about $4-5 \mathrm{~mm}$ in length. Consequently, the chewing plane rises up to the right and is $4-5 \mathrm{~mm}$ closer to the right orbital than to the left one. The resulting head posture (NHP) shows a persistent lateroflection and rotation. The mandible consequently hangs in an angulation, shifted to the right hand side.

\section{Ad 3: Asymmetries in shape and space of the prosthesis may force the tongue and the mandible into an unnatural position}

The third parameter derives from asymmetries in shape and space of (tongue!) the prosthesis. An (asymmetric) shape and space may force

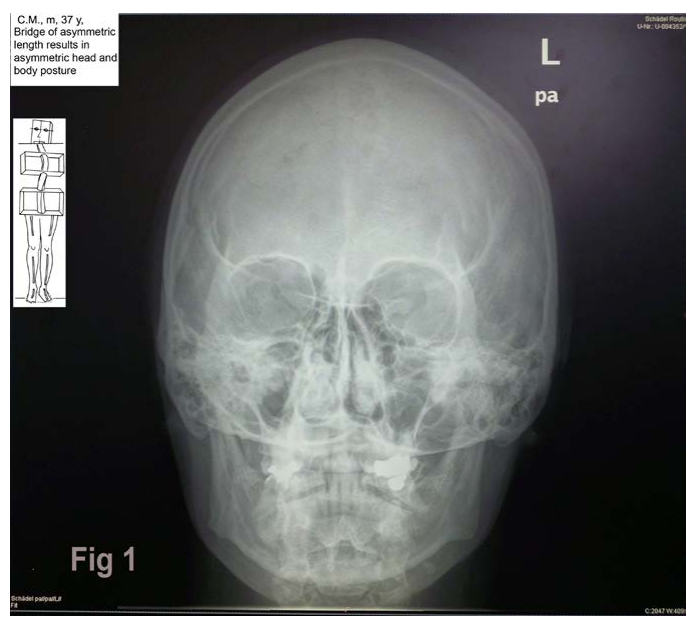

Figure 1. X-ray. The skull is positioned in lateroflection to the left and rotation to the right hand side. This hypothetically is caused by a lack in length in the bridge of about $3 \mathrm{~mm}$ in the upper left (II) quarter, which makes the chewing plane run more cranial (distance chewing plane - orbital is smaller in the left than in the right hand side). The mandible hangs at an angle to the skull, the left ramus is partially hidden in the skull. The left condyle is shifted dorsal-cranial, the right one ventral-caudal in relation to the fossa.

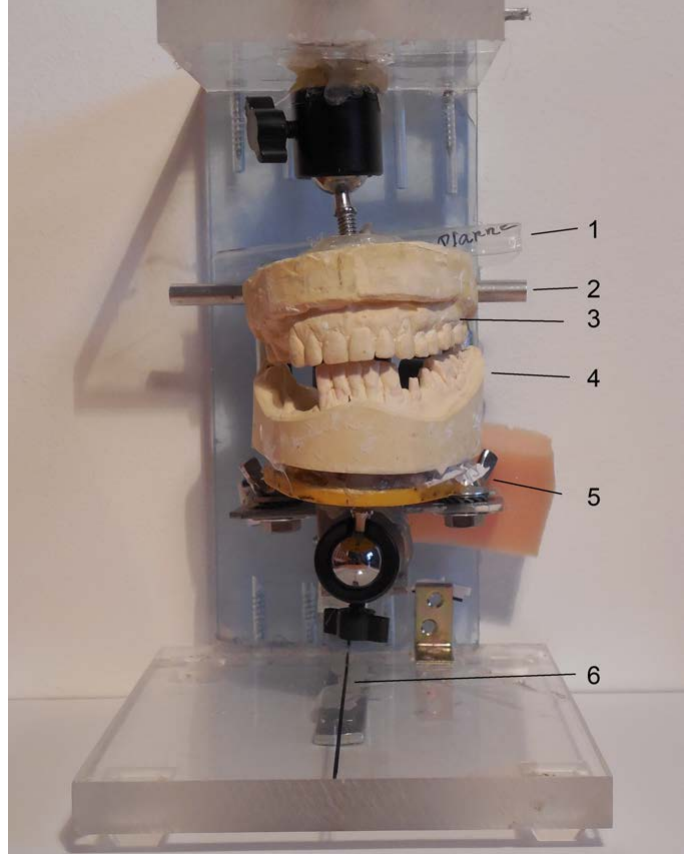

Figure 2. Spatial articulator, positioning of the casts according to the spatial position of teeth in the upright individual. Here: lateroflexion to the right, mandible along gravity. 1: axis of the fossae of TMJ; 2: axis of the condyles; 3: Upper cast with missing teeth and risen chewing plane in the right; 4: lower cast; 5 : holder for lower cast, 6 : sagittal line representing the movement direction,

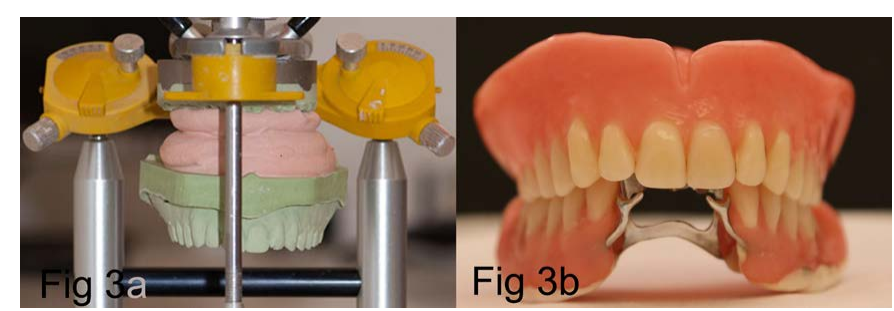

Figure 3. 3a) Position of upper cast in an SAM-articulator, according to registration in 30 dorsal position. 3b) upper and lower cast in the registered position.

the position of tongue and mandible. If such prosthesis is worn with registration, these mistakes will be imported into the future prosthesis.

The last sample patient suffers from an anterior disc displacement (ADD) with a clicking noise while opening the mouth. The presentation in the conventional articulator looks well (Figure 3a). It shows the symmetrically registrated position. In this view, there is no hint on the mechanism of an anterior disc dislocation in the right side.

The prosthesis (Figure $3 \mathrm{~b}$ ) shows an occlusion, again, there is no cause for a joint disorder.

In vivo, the patient's posture is but not upright (Figure $4 \mathrm{a} \& 4 \mathrm{~b}$ ) and the patient's head and body posture shows lateroflections and rotations. As an alternative, the position of teeth is shown in a spatial articulator, according to the way they are positioned in daily living.

\section{Positioning the casts in the spatial articulator}

1. In vivo: a marking cross in vivo is fixed to the upper prosthesis (Figure $4 \mathrm{a}$, horizontal line (1)). The transverse wing of the marking cross is aligned to the true horizontal, the forward wing to the movement direction.

2. Transfer: The marking cross (Figure $3 b$ ) is transferred with a 


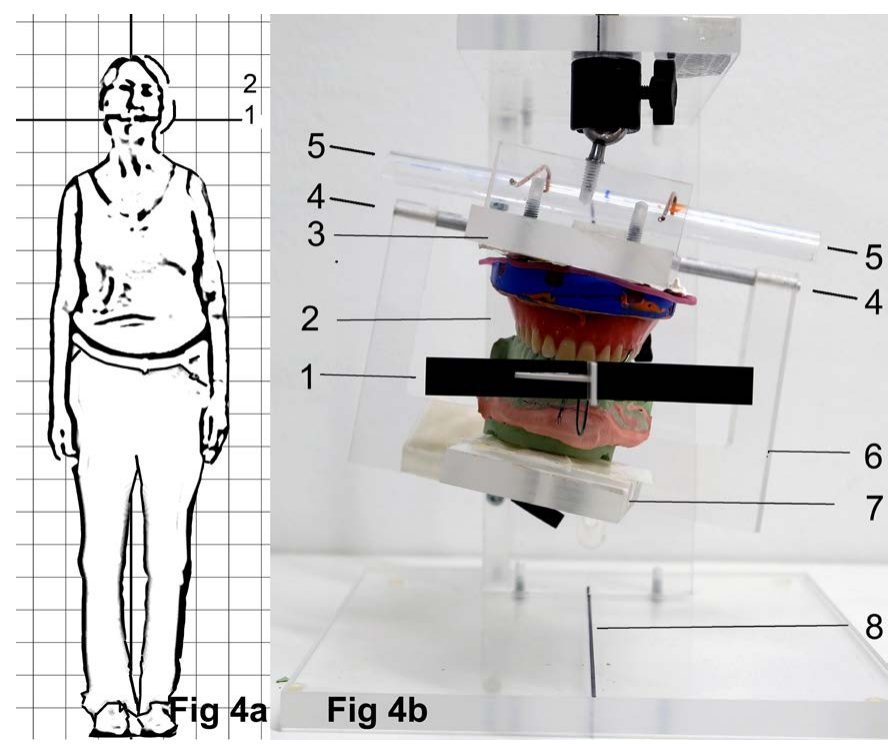

Figure 4. 4a) Marking cross in vivo, fixed to the upper prosthesis, 2: head in lateroflection to the left hand side. 4b) Spatial articulator; 1: marking cross in vitro, transferred with the upper prosthesis: the horizontal, black wing is aligned to transversal marks in the articulator, the white wing of the movement direction is aligned to the sagittal line (8). The Fossa axis (5) ("Ear axis") has been fixed to upper the upper cast by conventional registration (face bow). The condyle axis (4) is related to the lower cast. 2: prosthesis, according to lateroflection to the left; 3 : holder of upper prosthesis; 4: condyle axis; 5 : axis of the fossae; 6 : ramus; 7 : holder of lower prosthesis.

wafer from the patient to the upper cast.

3. In vitro, skull-related positioning: According to a registration with a face bow, the upper cast is fixed to the axis of the fossae of the TMJ.

4. In vitro, spatial related positioning: Afterwards, the upper prosthesis/axis of fossae is positioned in the spatial articulator (Figure $4 \mathrm{~b}$ ), so that the marking cross runs accordingly to the true horizontal and the sagittal wing points along the sagittal axis of the articulator.

The situation in the spatial articulator explains the mechanism of the ADD in combination with a manual examination: with closed mouth, the mandible is not able to overcome the right dental row and therefore stays on the right. The right condyle is risen with the right head side and additionally shifted lateral, dorsal and cranial to the fossa. The left condyle, in the lower side of the head is shifted dorsalcranial to the fossa.

Opening the mouth, the right condyle describes a huge bow moving downwards and to the left. It starts from the cranial-dorsallateral position and follows the course of the mandible along the gravitational direction. This movement exceeds the range of motion of the joint. It is not supported or guided by soft or hard tissue. This leads to habitual luxations of condyle and disc. The prosthesis turned out to limit the tongue and the mandible due to its shape. The risk of importing mistakes like these through registration is immense.

Self-Test/ Test: After having watched/ photographed the initial posture, the relationship between the course of upper chewing plane and posture may be tested by by placing a 3-4 $\mathrm{mm}$-stripe of cardboard between one dental row only or inserting either a wafer of asymmetric thickness to the upper teeth.

Alteration of head and body posture may be detected within 2 minutes. Photos may be taken before and after the change [14].

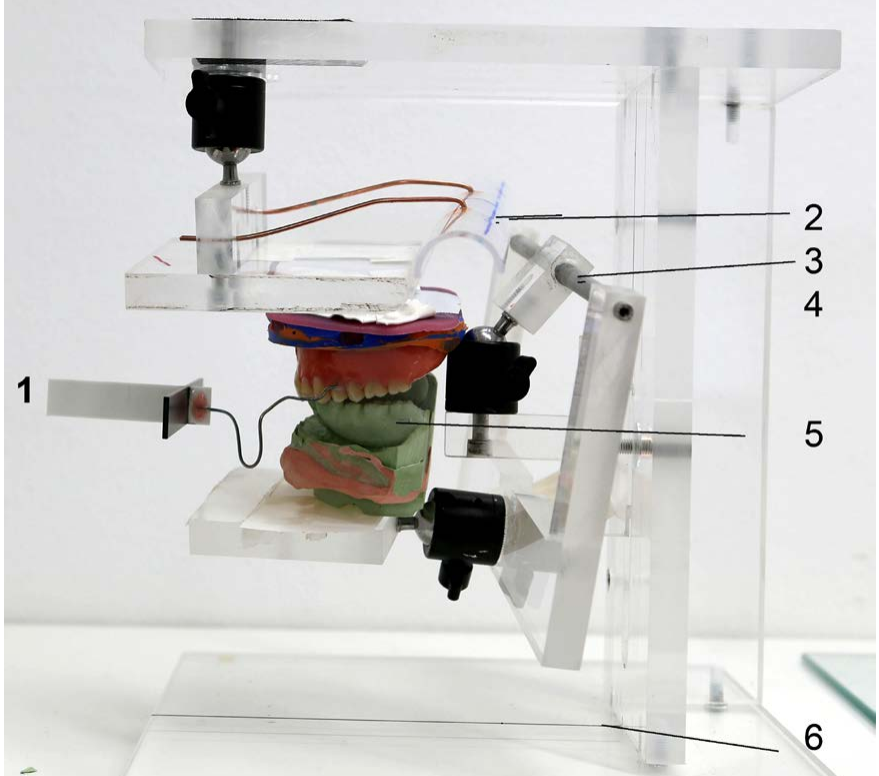

Fig 5

Figure 5. This view shows the position during orientation in the environment- it is discrepant to the registrated situation (Figures $3 a$ and $3 b$ ).

\section{Practical advice}

Before and after making a crown, a bridge or a whole prosthesis it is recommended to take a photo (whole body and head: front and profile; additionally one frontal portrait smiling with open mouth and visible edge of the upper teeth). If head and body posture is already lateroflected and rotated, you may test to level it out by putting $3 \mathrm{~mm}$ of cardboard in between the dental row in the side the head is flected to.

If the photo afterwards shows a worse posture-> level out the occlusal plane correctly [14].

\section{Conclusion}

The position of jaws is not well described by "occlusion". Additionally, upper and lower jaws seem to process spatial parameters. This may explain arising discrepancies in prostheses that are produced without considering the spatial relation of the jaws. The idea that jaws and teeth may be aligned to extracorporeal, spatial relations, has not been dealt with before. However, It offers a complete new point of view concerning dentistry, neurology and orthopedics. To prove these hypotheses and, if applicable, use them in dentistry and medicine, they have to be investigated first. If the upper chewing plane was a reference plane for spatial orientation, an alteration of its level (teeth length) will cause severe problems ("Temporomandibular dysfunction"). As teeth length is not conserved in dentistry so far, the involvement of dentists in these disorders seems to be nearby.

Investigation is recommended concerning the following hypotheses: 1. Does the maxilla and its teeth represent a spatial reference that during motion is aligned to the true horizontal and the movement direction? 2 . Will a wafer of asymmetric thickness change posture in order to realign the undersurface? 3. Is this independent on occlusion, e.g. does this work even without tooth contact in 3 minutes?

\section{Conflicts of interest}

The author declares that there is no conflict of interest. 


\section{Note}

The author is looking for a cooperation to examine the hypotheses, preferably 3D gait analysis. Please contact "mailto:ruth.nebel@mail.de" ruth.nebel@mail.de.

\section{References}

1. Nebel R (2014)Einfluss von Zahnveränderungen auf die Körperhaltung. Man Medizin 52: $390-401$.

2. Kovero O, Hurmerinta K, Zepa I, Huggare J, Nissinen M, et al. (2002) Maximal bite force and its associations with spinal posture and craniofacial morphology in young adults. Acta Odontol Scand 60: 365-369.[Crossref]

3. Morningstar MW, Pettibon BR, Schlappi H, Schlappi M, Ireland T V (2005) Reflex control of the spine and posture: a review of the literature from a chiropractic perspective. Chiropr Osteopat 13:16.

4. Salonen MA, Raustia AM, Huggare J (1993) Head and cervical spine postures in complete denture wearers. Cranio 11: 30-33.[Crossref]

5. Makofsky HW, Sexton TR, Diamond DZ (1991) State University of New York Stony. The effect of head posture on muscle contact position using the T-Scan system of occlusal analysis. New York: Cranio; 9:316-321.

6. Huggare JA, Raustia AM (1992) Head posture and cervicovertebral and craniofacial morphology in patients with craniomandibular dysfunction. Cranio 10: 173-177. [Crossref]
7. Huggare J (1991) Association between morphology of the first cervical vertebra, head posture, and craniofacial structures. Eur J Orthod 13: 435-440.[Crossref]

8. Matheus RA, Ramos-Perez FM, Menezes AV, Ambrosano GM, Haiter-Neto F, et al (2009) The relationship between temporomandibular dysfunction and head and cervical posture. J Appl Oral Sci 17: 204-208.

9. Arntsen T, Sonnesen L (2011) Cervical vertebral column morphology related to craniofacial morphology and head posture in preorthodontic children with Class II malocclusion and horizontal maxillary overjet. Am J Orthod Dentofacial Orthop 140: e1-e7. [Crossref]

10. Huang P, Zhang D (2012) Condylar position and condylar symmetry in patients with temporomandibular disorders. Shanghai Kou Qiang Yi Xue 21: 663-667. [Crossref]

11. Padala S, Padmanabhan S, Chithranjan AB (2015) Comparative evaluation of condylar position in symptomatic (TMJ dysfunction) and asymptomatic individuals. Indian $J$ Dent Res 23: 122. [Crossref]

12. Rinchuse DJ, Kandasamy S (2006) Centric relation: A historical and contemporary orthodontic perspective. J Am Dent Assoc 137: 494-501.[crossref]

13. Sener S, Akgunlu F (2011) Correlation between the condyle position and intraextraarticular clinical findings of temporomandibular dysfunction. Eur J Dent 5: 354 360. [crossref]

14. Nebel R (2016) Iatrogene Zahnlängenänderungen - Ursächlichfürsogenannte „CMD“? Teil 2. Dent Barom 4:24-27.

Copyright: (C2017 Nebel R. This is an open-access article distributed under the terms of the Creative Commons Attribution License, which permits unrestricted use, distribution, and reproduction in any medium, provided the original author and source are credited. 